\title{
Anotação semântica automática de Objetos de Aprendizagem Digitais: Um mapeamento sistemático de literatura
}

\author{
Leandro M. P. Sanches ${ }^{1}$, Laécio A. Costa ${ }^{1,2}$, Laís N. Salvador ${ }^{1}$, Marlo V. S. Souza ${ }^{1}$ \\ ${ }^{1}$ Programa de Pós-graduação em Ciência da Computação (PGCOMP) \\ Universidade Federal da Bahia (UFBA) - Salvador, BA - Brasil \\ ${ }^{2}$ Colegiado de Computação - Instituto Federal do Sertão Pernambucano (IFSertão) \\ BR 407, Km 08 - 56.314-520 - Petrolina, PE - Brasil \\ leandrompsanches@gmail.com, laecio.costa@ifsertao-pe.edu.br, \\ laisnseufba.br, marlo@dcc.ufba.br
}

\begin{abstract}
The Semantic Web technologies enable the semantic annotation of Learning Objects (LO), which allows more accurate and possibly quicker methods for the search and retrieval of LO. Since the LO manual semantic annotation a high-cost and mentally demanding task, this paper systematically mapped the methods for automatic semantic annotation of $L O$, as well the ontologies and metadata standards used in that process. A total of 466 papers were found and 13 papers were selected. The low number of articles presented in the final selection, approximately $2.8 \%$ in relation to the initial collection, may be indicate that there is still a need for further studies and experiments in this area of research.
\end{abstract}

Resumo. As tecnologias para Web Semântica possibilitam a anotação semântica de Objetos de Aprendizagem (OA) auxiliando na sua busca de forma mais precisa e rápida. Como o processo de anotação semântica manual de OA é uma tarefa dispendiosa, o presente trabalho mapeou sistematicamente os métodos para fazer a anotação semântica automática de $O A$, como também as ontologias e os padrões de metadados utilizados nesse processo. Foram encontrados 466 estudos e selecionados 13 artigos. A baixa quantidade de artigos apresentados na seleção final, aproximadamente 2,8\% em relação à coleta, pode ser um indício de que ainda há a necessidade de mais estudos e experimentos nessa área de pesquisa.

\section{Introdução}

Os Objetos de Aprendizagem (OA) são quaisquer recursos digitais que possam ser reutilizados para oferecer suporte à aprendizagem [Wiley 2000]. Esses recursos vêm sendo amplamente utilizados na área da educação [Müller 2015] e são considerados a forma mais adequada de desenvolver conteúdos de e-Learning, pois possibilitam a reutilização e a interoperabilidade de conteúdo educacional [Gonçalves 2005]. Contudo, para Tarouco e Schmitt (2010) o rápido aumento da quantidade e a baixa organização no armazenamento dos OA são fatores que dificultam a pesquisa, a recuperação e o reuso desses recursos.

\footnotetext{
"Projeto parcialmente financiado pela Fundação de Amparo à Pesquisa do Estado da Bahia (FAPESB).
} 
Para que um OA seja facilmente encontrado e, por conseguinte, mais bem aproveitado, ele precisa de metadados bem definidos que o descrevam. Os metadados são como tags (etiquetas) que classificam e categorizam a informação, melhorando, assim, a sua acessibilidade [Kawase et al. 2013, Miranda e Ritrovato 2014].

No entanto, para Kadar e Muntean (2014) as formas atuais de indexação para a Web não são suficientes para anotação de Objetos de Aprendizagem, uma vez que a indexação automática e os buscadores de conteúdo se restringem a aspectos sintáticos e que a indexação por especialistas humanos implica em altos custos. Nesse contexto, as abordagens baseadas nas tecnologias para Web Semântica podem oferecer soluções promissoras.

As tecnologias para Web Semântica visam obter dados estruturados em um formato padrão. No contexto educacional, essas tecnologias fornecem as ferramentas para a adição de metadados semânticos a OA, isto é, metadados compreensíveis por máquina e com significado semanticamente explícito. Tais metadados auxiliam na sua busca de forma mais precisa e rápida e possibilitam a descoberta e a reutilização inteligente de Objetos de Aprendizagem [Gonçalves e Carrapatoso, 2005].

Entretanto, a anotação de metadados semânticos, quando feita manualmente, é uma tarefa complexa, demorada e muitas vezes realizada de forma dependente de ontologias de domínios específicos necessitando ser refeita se o OA for utilizado em novos contextos [Farhat, Jebali e Jemni, 2015]. Além disso, segundo Repp e Meinel (2008), a anotação semântica é uma tarefa subjetiva e deve ser feita com cuidado.

Nesse contexto, identificadas a necessidade de adicionar semântica aos Objetos de Aprendizagem e as dificuldades da realização do processo de anotação semântica de forma manual, o presente trabalho apresenta um mapeamento sistemático de literatura (MSL) para encontrar os métodos e abordagens desenvolvidos para a anotação semântica automática dos Objetos de Aprendizagem. Adicionalmente, serão levantados as ontologias e os padrões de metadados utilizados nesse processo.

O restante do trabalho é organizado da seguinte forma: a Seção 2 traz metodologia utilizada no mapeamento sistemático e a Seção 3 discute e sintetiza os resultados. Por último, na Seção 4 são apresentadas as considerações finais.

\section{Metodologia do Mapeamento Sistemático}

A metodologia do presente artigo é baseada no modelo exposto por Kitchenham (2007), cuja condução é dividida em três etapas: 1) Planejamento da busca; 2) Execução da busca e 3) Documentação da busca. A etapa de planejamento inclui a definição das questões de pesquisa, estratégias de busca e critérios de seleção. A execução da busca contempla o levantamento e a seleção dos trabalhos, assim como, a extração e a análise dos dados obtidos. Na ultima etapa deve ser realizada a escrita e publicação do estudo.

\subsection{Questões de pesquisa}

As investigações sistemáticas de literatura requerem questões de pesquisa bem formuladas e claras [Sampaio e Mancini 2007]. Esse mapeamento visa levantar estudos que possam responder as seguintes questões de pesquisa: 1) "Quais os métodos existentes na literatura para fazer a anotação semântica automática de Objetos de Aprendizagem?"; 2) "Quais os padrões de metadados utilizados na anotação semântica 
VI Congresso Brasileiro de Informática na Educação (CBIE 2017)

Anais do XXVIII Simpósio Brasileiro de Informática na Educação (SBIE 2017)

automática de Objetos de Aprendizagem?" e 3) "Quais os tipos de ontologias utilizadas na anotação semântica automática de Objetos de Aprendizagem?".

\subsection{Estratégia de busca}

Para encontrar o maior número possível de estudos, um MSL utiliza uma estratégia de busca bem definida e clara, incluindo expressões de busca compostas por palavraschave, relacionadas com as questões de pesquisa, e por lógica booleana [Kitchenham 2007]. Nesse estudo, definiu-se a seguinte expressão de busca: "learning object" AND automatic AND ("semantic annotation" OR "metadata extraction").

Essa expressão de busca foi executada, no dia 07 de abril de 2017, em cinco ferramentas de busca selecionadas de acordo com a análise qualitativa de Buchinger, Cavalcanti e Hounsell (2014): IEEE Xplore, ACM Digital Library, Science Direct, Springer Link e Scopus.

\subsection{Critérios de Inclusão e Exclusão}

Nesse estudo ficaram definidos os seguintes critérios de inclusão: 1) Artigos primários publicados integralmente; 2) Trabalhos sobre a anotação semântica automática de Objetos de Aprendizagem; 3) Estudos publicados em inglês e 4). Em caso de estudos publicados em periódicos diferentes foi considerado o mais recente. Também ficaram definidos os seguintes critérios de exclusão: 1) Artigos que não focam no objeto desta pesquisa ou que não estão no domínio educacional; 2) Artigos duplicados e 3) Estudos inacessíveis. Foram aceitos somente trabalhos que tenham satisfeito todos os critérios de inclusão e não tenha satisfeito qualquer critério de exclusão.

\subsection{Processo de seleção dos trabalhos}

O processo de seleção dos estudos relevantes baseou-se no modelo de divisão em rodadas e subdivisão em etapas, proposto por Costa e Salvador (2015) e apresentado na Tabela 1. Na seleção primária realiza-se a leitura do título, resumo e palavras-chave, enquanto que na seleção secundária tem-se a leitura da introdução e da conclusão.

Tabela 1 - Rodadas de seleção

\begin{tabular}{|l|l|l|}
\hline \multicolumn{1}{|c|}{ Seleção } & \multicolumn{1}{|c|}{ Rodadas } & \multicolumn{1}{c|}{ Descrição } \\
\hline \multirow{3}{*}{ Primária } & $1^{\mathrm{a}}$ Rodada & Verificação de artigos primários \\
\cline { 2 - 3 } & $2^{\mathrm{a}}$ Rodada & Verificação de artigos duplicados ou atualizados \\
\cline { 2 - 3 } & $3^{\mathrm{a}}$ Rodada & Análise de Título, Abstract e Palavras-chave \\
\hline \multirow{2}{*}{ Secundária } & $4^{\mathrm{a}}$ Rodada & Verificação do idioma e da disponibilidade do estudo \\
\cline { 2 - 3 } & $5^{\mathrm{a}}$ Rodada & Análise da introdução e conclusão \\
\hline
\end{tabular}

Fonte: Adaptado de Costa e Salvador (2015)

\subsection{Ameaças à validade do mapeamento}

Se não realizadas com cuidado a seleção das palavras-chave e a construção da expressão de busca representam uma ameaça à validade do MSL. A primeira versão da expressão de busca continha o termo metadata generator, porém durante a execução da busca e a seleção dos artigos percebeu-se que o termo metadata extraction retornava um número maior de estudos sem ocorrer a perda de trabalhos relevantes. Portanto, foi realizada a substituição dos termos, resultando na expressão de busca apresentada na sessão 2. 
VI Congresso Brasileiro de Informática na Educação (CBIE 2017)

Anais do XXVIII Simpósio Brasileiro de Informática na Educação (SBIE 2017)

\section{Resultados e análises de dados}

Após a execução da expressão de busca foram encontrados 466 estudos. Ao término da seleção primária foram aceitos 89 estudos e após a seleção secundária foram aceitos 13 artigos. Entre os trabalhos rejeitados 33 textos foram considerados duplicados ou sofreram atualização, 29 estudos não eram artigos primários ou não estavam em inglês e 391 artigos não diziam respeito à anotação semântica automática de OA. A Tabela 2 resume a distribuição dos resultados de cada estapa da seleção.

Para responder a primeira questão de pesquisa os trabalhos aceitos foram classificados através de três diferentes categorias de acordo com o tipo de OA anotado: textual, multimídia e hipermídia. Assim, quatro estudos anotam OA no formato textual, cinco trabalhos abordam o formato multimídia, três artigos exploram o formato hipermídia e um estudo anota qualquer tipo de OA. É importante ressaltar que não foram aplicados filtros em relação ao tipo de OA. Os estudos aceitos são descritos e comparados na subseção 3.1 desse trabalho.

Tabela 2 - Resultados da etapa de execução

\begin{tabular}{|l|l|l|l|}
\hline \multicolumn{1}{|c|}{ Ferramenta de busca } & \multicolumn{1}{c|}{$\begin{array}{c}\text { Estudos } \\
\text { encontrados }\end{array}$} & $\begin{array}{c}\text { Seleção } \\
\text { Primária }\end{array}$ & $\begin{array}{c}\text { Seleção } \\
\text { secundária }\end{array}$ \\
\hline IEEE Xplore & $263(56,4 \%)$ & $53(59,5 \%)$ & $3(23,1 \%)$ \\
\hline Springer Link & $144(30,9 \%)$ & $28(31,5 \%)$ & $4(30,8 \%)$ \\
\hline Science Direct & $34(7,3 \%)$ & $3(3,4 \%)$ & $1(7,7 \%)$ \\
\hline Scopus & $20(4,3 \%)$ & $5(5,6 \%)$ & $5(38,4 \%)$ \\
\hline ACM Digital Library & $5(1,1 \%)$ & 0 & 0 \\
\hline Total & $\mathbf{4 6 6}$ & $\mathbf{8 9}$ & $\mathbf{1 3}$ \\
\hline
\end{tabular}

Em relação à segunda questão de pesquisa, constatou-se que aproximadamente $69 \%$ dos artigos aceitos não citam a utilização de padrões de metadados e os demais utilizam o padrão Learning Object Metadata (LOM) ${ }^{1}$, sendo que um único artigo utiliza o padrão MPEG-7 (Multimedia Content Description Interface) ${ }^{2}$ juntamente com o LOM.

A terceira questão de pesquisa está relacionada aos tipos de ontologias usadas na anotação, para respondê-la foram utilizadas duas formas de classificação de ontologias. A primeira, proposta por Guarino (1998), classifica as ontologias em quatro grupos de acordo com a generalidade: ontologia de nível superior, ontologia de domínio, ontologia de tarefas e ontologia de aplicação. A segunda classificação, proposta por Guarino e Welty (2000), realiza a divisão em quatro categorias de acordo com a profundidade ontológica: vocabulário, taxonomia, sistema relacional e teoria axiomática completa.

Alguns estudos não descrevem completamente, nomeiam ou classificam as ontologias dificultando a determinação da profundidade ontológica. Nesses casos foi considerada a classificação mínima aplicável, por exemplo: se um artigo apresentar uma ontologia com relações hierárquicas e não hierárquicas, porém não possibilitar a determinação da existência de axiomas, essa será classificada como sistema relacional.

\footnotetext{
${ }^{1}$ Padrão de metadados específico para Objetos de Aprendizagem proposto pelo IEEE (2002).

${ }^{2}$ Padrão de descrição de conteúdo multimídia ISO/IEC 15938.
} 
Dos estudos aceitos, aproximadamente 85\% (11 artigos) utilizam estruturas ontológicas. Em relação à generalidade desses recursos, todos os 11 artigos utilizam pelo menos uma ontologia de domínio, sendo que, dois desses também utilizam ontologia(s) de nível superior. Não foram encontrados estudos com ontologias de aplicação ou de tarefas. Em relação à profundidade ontológica desses recursos, foram encontrados sete sistemas relacionais, cinco taxonomias, um sistema axiomático completo e um vocabulário. Além disso, dois artigos apresentaram propostas genéricas para serem utilizadas com qualquer ontologia de domínio, logo impossibilitando a determinação da profundidade ontológica.

\subsection{Análise descritiva dos métodos encontrados}

Os trabalhos apresentados por Reinhardt et al. (2007); Yu et al. (2007); Jain, Pareek (2010) e Smine, Faiz e Desclés (2013), abordam diferentes métodos de anotação semântica de OA no formato textual. A proposta de Reinhardt et al. (2007) apresenta dois módulos: a extração de metadados e a análise semântica. Essa proposta tem como saída um arquivo em XML (eXtensible Markup Language) no padrão LOM. Embora o processo de extração seja automático, ainda é possível ao autor do OA realizar edições ao final do processo.

A abordagem de Yu et al. (2007) utiliza técnicas de Reconhecimento de Entidade Nomeada (REN), segmentação de trechos baseado em tópico e associação dos termos com uma taxonomia sobre o domínio de ementas de cursos (syllabus, em inglês), para anotar semanticamente os conteúdos. O REN é realizado em paralelo com a segmentação e objetiva reconhecer as entidades como pessoas, datas, locais, organizações e códigos de curso. A segmentação objetiva reconhecer os limites de cada tópico no documento analisado. Os autores utilizam a taxonomia para auxiliar na reconciliação de diferentes vocabulários.

A proposta de Jain e Pareek (2010) é identificar conceitos de diferentes áreas temáticas no mesmo OA e comparar a relevância dos conceitos identificados com o auxílio de uma taxonomia descrevendo o domínio de diferentes disciplinas. O estudo trata da extração dos principais conceitos presentes em OA textuais utilizando a taxonomia e faz parte do projeto de desenvolvimento de um Web Service para anotação automática de OA.

O trabalho de Smine, Faiz e Desclés (2013) apresenta um modelo de anotação semântica automática de OA textuais. A proposta é realizada de acordo com um sistema relacional de categorias de OA (definição, exemplo, exercício, etc.). O processo de anotação ocorre em três etapas: 1) pré-processamento: os documentos originais são convertidos para o formato de texto plano; 2) segmentação: com a análise de indicadores tipográficos e bases linguísticas é possível determinar a borda de uma sentença e 3) anotação semântica: nesta etapa o algoritmo anota os segmentos de texto de acordo com as categorias semânticas, através da comparação dos termos presentes no segmento com regras linguísticas específicas de cada categoria.

Os autores Fogarolli e Ronchetti (2008) e Repp, Linckels e Meinel (2008) realizam a anotação semântica de Objetos de Aprendizagem multimídia, vídeos de aulas/palestras, utilizando como fontes de informação a transcrição da camada de áudio em texto e o conteúdo textual dos slides do professor/palestrante. 
A partir dos dados extraídos, Fogarolli e Ronchetti (2008) aplicam a ferramenta Lucene $^{3}$ para extrair e classificar os termos relevantes. Em contrapartida, Repp, Linckels e Meinel (2008) realizam a remoção de termos contextualmente irrelevantes, a lematização ${ }^{4}$ e a classificação dos termos relevantes através de um algoritmo próprio baseado na frequência que um termo ocorre dentro de um OA em comparação com os outros OA.

Posteriormente, Fogarolli e Ronchetti (2008), aproveitam a estrutura taxonômica da Wikipédia e associam os termos com melhor classificação às diferentes páginas dessa enciclopédia digital. $\mathrm{O}$ algoritmo também faz uso do conteúdo multilíngue da Wikipédia e, caso necessário, de um tradutor para anotar termos de diferentes idiomas. Já em Repp, Linckels e Meinel (2008) os OA são anotados utilizando lógica de descrição gerada através do mapeamento dos termos mais bem classificados em relação aos conceitos presentes em uma ontologia de domínio (sistema axiomático completo) sobre redes de computadores. Um dicionário de sinônimos é aplicado para desambiguação e para proporcionar certa tolerância a termos transcritos erroneamente.

Os autores Bolettieri et al. (2007) apresentam uma proposta para anotar OA multimídia: vídeos e apresentações de slides. Os vídeos são segmentados através de um algoritmo de detecção de limites e são determinados os quadros principais de cada segmento. O sistema anota os segmentos com metadados no padrão MPEG-7. Cada metadado contém a transcrição da camada de áudio, o texto dos quadros e os diferentes descritores visuais MPEG-7. A anotação de metadados de apresentações de slides é realizada extraindo o título e o conteúdo textual de cada slide. As informações extraídas são organizadas através do padrão MPEG-7, pois esse suporta a decomposição da apresentação completa em slides individuais. Os metadados MPEG-7 gerados automaticamente nesse estudo são codificados em XML e armazenados juntamente com metadados anotados manualmente no padrão LOM.

Os autores Jovanovic, Gasevic e Devedzic (2006) também anotam semanticamente Objetos de Aprendizagem multimídia. Esse estudo anota de modo automático os fragmentos de apresentações de slides e de modo semi-automático as apresentações de slides completas, utilizando ontologias de domínio e o padrão LOM. O sistema tem como entrada as apresentações de slides com metadados LOM anotados manualmente. Uma ontologia de domínio (sistema relacional) descrevendo a estrutura da apresentação de slides é utilizada para fragmentá-la. A anotação dos fragmentos é realizada através da derivação dos metadados atribuídos à apresentação de slides completa, de técnicas de mineração do texto e da análise da organização estrutural do fragmento. Os metadados extraídos são armazenados no padrão LOM com codificação em RDF (Resource Description Framework) e são associados a outras duas ontologias de domínio: uma taxonomia sobre as funções pedagógicas dos OA e um sistema relacional sobre conceitos específicos da área de servidores web.

Os autores, Zhang e Shen (2009), anotam qualquer OA multimídia através de uma rede semântica composta por OA associados a palavras-chave. Cada ligação entre um OA e uma palavra-chave possui um peso que é modificado conforme feedbacks recebidos dos usuários durante a busca dos OA. O sistema de pesos possibilita a adição

\footnotetext{
${ }^{3}$ Biblioteca de software para pesquisa e indexação de texto disponível em: <http://lucene.apache.org/>.

${ }^{4}$ Determinação da forma canónica da palavra, desconsiderando gênero e número.
} 
e a remoção de palavras-chave. O mapeamento das relações entre as palavras-chave e sua desambiguação é realizado através da ontologia de nível superior WordNet $^{5}$.

Três estudos anotam semanticamente OA hipermídia de modos diferentes: Pipitone e Pirrone (2012), Atkinson et al. (2013) e Vidal et al. (2014), sendo que os dois primeiros focam em páginas web e o último em livros interativos codificados em XML.

Em Atkinson et al. (2013) é descrita uma abordagem para identificar e anotar OA em páginas de internet através da combinação de aprendizagem de máquina com Processamento de Linguagem Natural (PLN). O processo começa com a pesquisa realizada pelo usuário em um rastreador de propósito específico (crawler, em inglês). Esse rastreador coleta primeiro as páginas mais promissoras com base em sementes geradas anteriormente. Um extrator de metadados obtém informações das páginas recuperadas e um indexador semântico avalia e alimenta o rastreador utilizando os dados fornecidos pelo extrator. Para isso, as principais informações sobre os documentos são automaticamente identificadas e os hiperlinks são analisados de acordo com seu texto. Assim, é criada uma lista de tópicos contendo os pesos associados a cada página da Web ou hiperlink. A hierarquia dos temas presentes nessa lista foi extraída de uma taxonomia sobre o domínio de história e geografia.

No trabalho de Pipitone e Pirrone (2012) a anotação semântica é obtida através de processos de compreensão de linguagem natural realizados por um agente artificial. Nesse trabalho, os autores identificam instâncias de classes e suas relações através da busca por padrões em documentos textuais. Tais padrões são construídos a partir de uma ontologia de domínio, fornecida ao sistema como entrada, e de um conjunto de recursos lexicais, tais como as ontologias de nível superior WordNet e o FrameNet ${ }^{6}$. O sistema proposto nesse trabalho assume um caráter incremental, pois permite a utilização de diferentes ontologias de domínio processadas por agentes distintos. Cada agente adquire conhecimento com as anotações anteriores e anota o OA com novas informações.

O estudo de Vidal et al. (2014) utiliza a DBpedia ${ }^{7}$. O processo se inicia na análise do documento Web com a combinação de técnicas de PLN e de medidas de similaridade para extrair os termos relevantes classificados de acordo com a sua frequência e a sua localização na estrutura do documento. Para cada termo é obtida uma lista de instâncias da DBpedia candidatas para anota-lo. Para selecionar uma instância por termo utiliza-se um algoritmo de filtragem considerando o contexto do documento e essa inclusão de instâncias pode ser supervisionada por especialistas. Um (sub)grafo é gerado a partir de cada instância, o algoritmo filtra os termos relacionados à instância em questão e anota os mais relevantes. A relevância de um nó é dada pelo seu tipo de informação e pelas suas relações, considerando a importância dessa relação para o contexto analisado.

No estudo de Farhat, Jebali e Jemni (2015) metadados no padrão LOM são utilizados como entrada para o processo de anotação semântica automática de qualquer tipo de OA. A arquitetura proposta é dividida em três partes: 1) extração de conceitos e suas relações a partir de uma ontologia de domínio dada como entrada, 2) aplicação

\footnotetext{
${ }^{5}$ Sistema relacional léxico para a língua inglesa, disponível em: $<$ https://wordnet.princeton.edu/>.

${ }^{6}$ Sistema relacional léxico para a língua inglesa, disponível em: $<$ http://framenet.icsi.berkeley.edu/>.

${ }^{7}$ Base de dados (sistema relacional) abertos e interconectados disponível em $<$ http://dbpedia.org/>.
} 
VI Congresso Brasileiro de Informática na Educação (CBIE 2017)

Anais do XXVIII Simpósio Brasileiro de Informática na Educação (SBIE 2017)

técnicas de processamento para analisar e preparar os metadados LOM como fontes de informação e 3) extração de informações através de um algoritmo que aplica uma classificação com base na hierarquia da ontologia.

\section{Considerações finais}

Nesse trabalho foi realizado um mapeamento sistemático de literatura objetivando responder questões de pesquisa relacionadas aos métodos de anotação semântica automática de $\mathrm{OA}$, aos tipos de ontologias e aos padrões de metadados utilizados nesse processo, assim como, identificar lacunas de pesquisa.

A partir da seleção final de artigos, encontrados em fontes de referência amplamente consultadas pela comunidade de computação, foi possível verificar diversas propostas para anotar automaticamente metadados semânticos a OA textuais, multimídias e hipermídias.

Entre os estudos selecionados, Farhat, Jebali e Jemni (2015) afirmam que os metadados semânticos não necessariamente precisam substituir os metadados tradicionais. Deste modo, tanto Farhat, Jebali e Jemni (2015) quanto Jovanovic, Gasevic e Devedzic (2006) utilizam metadados no padrão LOM como entrada para o processo de anotação semântica. Seguindo essas propostas, um campo de pesquisa inexplorado é a anotação semântica a partir da utilização de outros padrões de metadados, entre eles o Dublin Core $(D C)$, que é o padrão adotado por um dos repositórios de Objetos de Aprendizagem mais utilizados, o DSpace.

Além disso, todos os trabalhos encontrados que anotam semanticamente OA no formato de vídeos obtêm informações a partir da transcrição da camada de áudio em texto. Assim, as técnicas propostas por Fogarolli e Ronchetti (2008) e por Repp, Linckels e Meinel (2008) podem ser estendidas para OA no formato de apenas áudio, pois nenhum trabalho encontrado realiza a anotação semântica de OA nesse formato.

Durante a execução deste mapeamento percebeu-se que, em alguns estudos, o processo de anotação semântica ainda necessita de alguma intervenção ou de supervisão humana (aproximadamente 38,5\%). Também foi possível notar que menos da metade dos trabalhos encontrados (aproximadamente 31\%) utilizam padrões de metadados e, quando esses são utilizados, há uma preferência pelo uso do padrão LOM.

A baixa taxa de utilização de padrões de metadados na anotação semântica possivelmente deve-se ao fato de que os padrões mais utilizados para Objetos de Aprendizagem, como o LOM, o Dublin Core e o IMS Metadata [Miranda e Ritrovato 2014], originalmente não preveem a descrição da semântica do conteúdo. O desenvolvimento e a adoção pela comunidade acadêmica de um padrão de metadados para OA voltado para a descrição semântica do conteúdo possivelmente reverteria esse quadro e pode caracterizar-se como uma lacuna de pesquisa.

Em relação às ontologias, observou-se uma predileção à utilização de ontologias de domínio, contudo também foram encontrados trabalhos com ontologias linguísticas e com ontologias de nível superior. A baixa quantidade de artigos apresentados na seleção final, aproximadamente $2,8 \%$ em relação à coleta, pode ser considerada como indício da necessidade de mais estudos e experimentos voltados para a anotação automática de metadados semânticos de Objetos de Aprendizagem. 
VI Congresso Brasileiro de Informática na Educação (CBIE 2017)

Anais do XXVIII Simpósio Brasileiro de Informática na Educação (SBIE 2017)

\section{Referências}

Atkinson, J.; Gonzalez, A.; Munoz, M.; Astudillo, H. (2013) Web Metadata Extraction and Semantic Indexing for Learning Objects Extraction. IEA/AIE. Lecture Notes in Computer Science, vol. 7906. Springer, Berlin, Heidelberg.

Buchinger, D.; Cavalcanti, G. A. D. S.; Hounsell, M. D. S. (2014) Mecanismos de busca acadêmica: uma análise quantitativa. Revista Brasileira de Computação Aplicada, v 6, n 1, p 108-120.

Bolettieri P.; Falchi, F.; Gennaro, C.; Rabitti, F. (2007) Automatic metadata extraction and indexing for reusing e-learning multimedia objects. Workshop on multimedia information retrieval on The many faces of multimedia semantics - May, $\mathrm{p} 21$.

Costa, L. A.; Salvador, L. N (2015). Ambiente de aprendizagem presencial e virtual integrados com a computação ubíqua: Um mapeamento sistemático da literatura. In: XX Congresso Internacional de Informática Educativa. Santiago: Universidade do Chile, v. 11, p. 211-220.

Farhat, R.; Jebali, B.; Jemni, M. (2015) Ontology based semantic metadata extraction system for learning objects. In: CHEN, G. et al. (Eds.). Emerging Issues in Smart Learning. Berlin, Heidelberg: Springer Berlin Heidelberg. p 247-250.

Fogarolli, A.; Ronchetti, M. (2008) Intelligent Mining and Indexing of Multi-language e-Learning Material. (Eds.). New Directions in Intelligent Interactive Multimedia. Berlin, Heidelberg: Springer Berlin Heidelberg, p 395-404.

Gonçalves, V. M. B. (2005) Ensino à distância e Web Semântica. Newsletter Professores Inovadores, Microsoft Educação, 6 ed. IPB.

Gonçalves, V. M. B.; Carrapatoso, E. M. (2005) Um sistema de e-learning para a Web Semântica baseado na tecnologia de agentes. In: Conferência IADIS IberoAmericana WWW/Internet, Lisboa.

Guarino, N. (1998) Formal Ontology and Information Systems. Proceedings of the first international conference, June, p 3-15.

Guarino, N.; Welty, C. (2000) Conceptual modeling and ontological analysis. The AAAI-2000, Disponível em: <http://www.cs.vassar.edu/faculty/welty/aaai-2000/>.

Jain, S.; Pareek, J. (2010) Automatic topic(s) identification from learning material: An ontological approach. 2nd ICCEA, v 2, p 358-362.

Jovanovic, J.; Gasevic, D.; Devedzic, V. (2006) Ontology-Based Automatic Annotation of Learning Content. International Journal on Semantic Web and Information Systems, v 2, n 2, p 91-119.

Kadar M.; Muntean M. A. (2014) Collaborative Environment for E-training in Archaeology. Agent and Multi-Agent Systems: Technologies and Applications. Advances in Intelligent Systems and Computing, v 296, p 303 - 312.

Kawase, R.; Sienhndel, P.; Nunes, B. P.; Fisichella, M. (2013). Automatic competence leveling of learning objects. Proceedings IEEE 13th ICALT, p 149-153.

Kitchenham, B. (2007). Guidelines for Performing Systematic Literature Reviews in Software Engineering. Technical Report EBSE, Keele University and University of Durham. 
VI Congresso Brasileiro de Informática na Educação (CBIE 2017)

Anais do XXVIII Simpósio Brasileiro de Informática na Educação (SBIE 2017)

Miranda, S.; Ritrovato, P. (2014) Automatic extraction of metadata from learning objects. Proceedings of International Conference on Intelligent Networking and Collaborative Systems, IEEE INCoS, p 704-709.

Müller, T. J. (2015). Objetos de Aprendizagem Multimodais e Ensino de Cálculo: Uma Proposta Baseada em Análise de Erros. 203 p. Tese (Doutorado em Informática na Educação) - Universidade Federal do Rio Grande do Sul, Porto Alegre.

Reinhardt, W.; Mletzko, C.; Schmidt, B.; Magenheim, J.; Schauerte, T. (2008) Knowledge Processing and Contextualisation by Automatical Metadata Extraction and Semantic Analysis. In: Dillenbourg P., Specht M. (eds) Times of Convergence.

Repp, S.; Linckels, S.; Meinel, C. (2008) Question answering from lecture videos based on an automatic semantic annotation. ACM SIGCSE Bulletin, v 40, n 3, p 17.

Sampaio, R. F.; Mancini, M. C. (2007) Estudos de revisão sistemática: um guia para síntese criteriosa da evidência científica. Brazilian Journal of Physical Therapy, v 11, n 1, p 83-89.

Smine, B.; Faiz, R.; Desclés, J.-P. (2013) Relevant learning objects extraction based on semantic annotation of documents. International Journal of Metadata Semantics and Ontologies, v 8, n 1, p 13-27.

Tarouco, L.; Schmitt, M. (2010). Adaptação de metadados para Repositórios de Objetos de Aprendizagem. Revista RENOTE: Novas Tecnologias na Educação, v 8, n 2.

Pipitone, A.; Pirrone, R. (2012) Cognitive Linguistics as the Underlying Framework for Semantic Annotation. 2012 IEEE Sixth International Conference on Semantic Computing, p 52-59.

The Institute of Electrical and Electronics Engineers - IEEE (2002). IEEE Standard for Learning Object Metadata. Disponível em: http://ieeexplore.ieee.org/servlet/opac?punumber=8032. Acesso 22 de Maio de 2017.

Vidal, J. C; Lama, M.; Otero-Gárcia, E.; Bugarin, A. (2014) Graph-based semantic annotation for enriching educational content with linked data. Knowledge-Based Systems, v 55, p 29-42.

Wiley, D. A. (2000) Connecting learning objects to instructional design theory: A definition, a metaphor, and a taxonomy. Disponível em: http://reusability.org./read/chapters/wiley.doc. Acesso em: 22 de Maio de 2017.

Yu, X.; Tungare, M.; Fan, W.; Pérez-Quiñones, M.; Fox, E.; Cameron, W.; Cassel, L. (2007) Using Automatic Metadata Extraction to Build a Structured Syllabus Repository. In: GOH, D. H.-L. et al. (Eds.). Asian Digital Libraries. Looking Back 10 Years and Forging New Frontiers. ICADL, Hanoi, Vietnam. p 337-346.

Zhang, T. Z.; Shen, R. M. (2009) Learning objects automatic semantic annotation by learner relevance feedback. Biomedical Engineering and Informatics. BMEI'09. 2nd International Conference on, p. 1-4. 